\title{
Theoretical investigation of fluorination effect on the charge carrier transport properties of fused anthra-tetrathiophene and its derivatives
}

\author{
Jun Yin, Kadali Chaitanya, Xue-Hai Ju*
}

Key Laboratory of Soft Chemistry and Functional Materials of MOE, School of Chemical Engineering, Nanjing University of Science and Technology, Nanjing 210094, P. R. China \begin{abstract}
The crystal structures of known anthra-tetrathiophene (ATT) and its three fluorinated derivatives (ATT1, ATT2 and ATT3) were predicted by the Monte Carlo-simulated annealing method with the embedded electrostatic potential (ESP) charges. The most stable crystal structures were
\end{abstract} further optimized by the density functional theory with the dispersion energy (DFT-D) method. In addition, the effect of the electron-withdrawing fluorine atoms on the molecular geometry, molecular stacking, electronic and transport properties of title compounds were investigated by the density functional theory and the incoherent charge-hopping model. The calculated results show that the introduction of fluorine atoms does not affect the molecular planarity but decreases the HOMO-LUMO gap, which is beneficial to electron injection and provides more charge carrier stabilization. The improved electron mobility from ATT to ATT3 is attributed to the favorable molecular packing with strong $\pi-\pi$ interaction and the short stacking distance. ATT2 and ATT3 exhibit remarkable angular dependence of mobilities and anisotropic behaviors. The band structures reveal that all the paths with larger transfer integrals are along the directions of large dispersions in the valence band (VB) and conduction band $(\mathrm{CB})$. ATT3 has the largest electron mobility $\left(0.48 \mathrm{~cm}^{2} \cdot \mathrm{V}^{-1} \cdot \mathrm{s}^{-1}\right)$ among the four

\footnotetext{
* Corresponding author. E-mail: xhju@ njust.edu.cn; Tel: +86 25 84315947-801; fax: +86 2584431622
} 
compounds, indicating that fluorination is an effective approach to improve electron transport.

Keywords: Crystal structure prediction; Density functional theory (DFT); Anthra-tetrathiophene derivatives; Electron mobility

\section{Introduction}

Recently organic semiconductors have played an important role in the field of electronic and optoelectronic materials owing to their low manufacturing costs, large-area coverage, mechanical flexibility and lightweight [1-3]. Generally, oligothiophenes and arene derivatives have shown high hole mobility and good air-stability due to their large $\pi$-conjugation and outstanding chemical and physical properties [4]. However, the development of ambient-stable n-type semiconductors has largely lagged behind that of p-type ones due to a lack of considerable electron mobility [5]. On account of this, past few years several research groups have been working on the design and synthesis of high-performance n-type $\pi$-conjugated organic semiconductors. Recently, Liu et al. devised a simple synthesis of two sulfur-rich anthra-[1,2-b:4,3-b':5,6-b":8,7-b'"']tetrathiophene-based heteroarenes (ATT) with favorable $\pi-\pi$ interactions [6]. In which, fused heteroarene unit, anthra-[1,2-b:4,3-b':5,6-b":8,7-b'"] tetrathiophene serves as the basic skeleton of $\pi$-extended planar systems and facilitates molecular self-association through $\pi$ - $\pi$ stacking. ATT has shown a good stability at ambient temperature due to its favorable overlap packing and large $\pi$-conjugation. Additionally, the sulfur-sulfur interactions, originating from the high polarizability of sulfur atom in thiophene rings, could strongly affect the solid-state packing of the compounds and determine the transport network [7, 8]. Over the past decades, experimental and theoretical investigations have shown that functionalizing p-type semiconductor with electron-withdrawing groups was a promising way to convert them into n-type ones [7,9]. Attaching 
electron-withdrawing substituents such as $\mathrm{Cl}, \mathrm{Br}$, and $\mathrm{F}$ to the $\pi$-conjugated ring can lower the molecular orbital energy level and provide stronger electron affinities, which will facilitate the injection of electrons $[10,11]$. Based on this, we design three ATT derivatives (ATT1, ATT2 and ATT3) with the strong electron-withdrawing fluorine substituents based on the precursor molecule ATT to shed light on molecular design for n-type organic semiconductor through simple chemical structural modification.

Motivated by the interesting fact that the slight modification of organic molecules could induce different optical and electrochemical performances, we investigated the transport properties of the fluorinated ATT derivatives based on the density functional theory (DFT) coupled with the incoherent charge-hopping model to explore the effect of the number and location of fluorine atoms on the charge transfer property of ATT. In this work, three fluorinated ATT molecules ATT1, ATT2 and ATT3 are optimized by the GGA/PBE functional. Then, the optimized molecules ATT1, ATT2 and ATT3 with electrostatic potential-fitted charge are simulated using the Monte Carlo-simulated annealing method based on the molecular mechanics (MM) to search for reasonable crystal structures. Afterwards, the predicted ATT1, ATT2 and ATT3 crystal structures with the lower energy were selected for further optimized calculations by the DFD-D method at the PBE level. A quantum chemistry and molecular mechanic combined approach is applied to investigate the geometries, molecular orbitals, electron affinities, ionization potentials and reorganization energies of the ATT derivatives. The intermolecular transfer integrals and charge transport mobilities at room temperature have been deduced with the predicted single crystal structures. This work is to elucidate the carrier transport behavior, and to formulate structure-property relationships and proceeding further with rational design of high-performance n-type organic semiconductors. 


\section{Theoretical and computational methodology}

\subsection{Theoretical methodology}

There are mainly two types of charge transport mechanisms in organic electronic materials at present: the incoherent hopping and the coherent band mechanism [12]. The charge transport is governed by hopping mechanism when the system is disordered at room temperature. According to hopping mechanism, each hopping step has been considered as a nonadiabatic electron-transfer reaction involving a self-exchange charge from a charged molecule to an adjacent neutral one. The rate of charge transfer between neighboring molecules, $k$, can be expressed by the standard Marcus equation in terms of the reorganization energy $\lambda$, the transfer integral $V$, and the temperature $T$ as [13]:

$$
k=\frac{2 \pi V^{2}}{h}\left(\frac{\pi}{\lambda k_{B} T}\right)^{\frac{1}{2}} \exp \left(\frac{\lambda}{4 k_{B} T}\right)
$$

where $h$ and $k_{B}$ are the Planck and Boltzmann constants, respectively.

The reorganization energy $\lambda$ consists of intramolecular reorganization energy and intermolecular reorganization energy. However, intermolecular reorganization energy is much smaller than intramolecular one in organic solid $[14,15]$. Herein, the intramolecular reorganization energy, which reflects the deformation of the molecular geometry in charge transfer process, is only considered in this study. Thus, the reorganization energy could be expressed by the adiabatic potential (AP) energy surface approach [16]:

$$
\lambda_{ \pm}=E_{0}\left(Q_{ \pm}\right)-E_{0}\left(Q_{0}\right)+E_{ \pm}\left(Q_{0}\right)-E_{ \pm}\left(Q_{ \pm}\right)
$$

where $E$ is energy and $Q$ is geometry. The subscripts $0,+$ and - denote neutral, cationic, and anionic states, respectively. For example, $E_{+}\left(Q_{0}\right)$ is the energy of cation with geometry of the optimized neutral molecule. Then, the ionic state properties such as vertical ionization potential $\left(I P_{V}\right)$, adiabatic ionization potential $\left(I P_{a}\right)$, vertical electron affinities $\left(E A_{V}\right)$, and adiabatic electron affinities $\left(E A_{a}\right)$ were calculated 
by the following formulas [17]:

$$
\begin{aligned}
& I P_{V} / I P_{a}=E_{+}\left(Q_{0}\right) / E_{+}\left(Q_{+}\right)-E_{0}\left(Q_{0}\right) \\
& E A_{V} / E A_{a}=E_{0}\left(Q_{0}\right)-E_{-}\left(Q_{0}\right) / E_{-}\left(Q_{-}\right)
\end{aligned}
$$

The evolution of transfer integral $V$ is based on the molecular orbitals of the conjugated molecules.

The coupling matrix element could be expressed by the site-energy corrected method [18]:

$$
V=\frac{J_{12}-\frac{1}{2}\left(E_{1}+E_{2}\right) S_{12}}{1-S_{12}^{2}}
$$

here, the transfer integral $V$ could be written with charge transfer integral $J$, overlap matrix $S$ and site energy of monomer $E$, respectively.

In the calculation of transfer integral in single crystal, the monomer orbitals with proper orthogonalization are used as basis sets for the Hamiltonian of the dimer system. For hole transport the highest occupied molecular orbitals (HOMOs) of monomers are used as the basis function while for electron transport the lowest unoccupied molecular orbitals (LUMOs) are used correspondingly, and then $S, J$ and $E$ could be expressed as [19]:

$$
\begin{aligned}
& J_{12}=\left\langle\varphi_{1}^{H / L}|h| \varphi_{2}{ }^{H / L}\right\rangle \\
& S_{12}=\left\langle\varphi_{1}^{H / L} \mid \varphi_{2}{ }^{H / L}\right\rangle \\
& E_{1}=\left\langle\varphi_{1}^{H / L}|h| \varphi_{1}^{H / L}\right\rangle \\
& E_{2}=\left\langle{\varphi_{2}}^{H / L}|h| \varphi_{2}{ }^{H / L}\right\rangle
\end{aligned}
$$

where $h$ is the Kohn-Sham Hamiltonian of a dimer and $\varphi_{1}{ }^{\mathrm{H} / \mathrm{L}}, \varphi_{2}{ }^{\mathrm{H} / \mathrm{L}}$ are the localized molecular orbitals of adjacent monomers.

Assuming that there is no correlation between charge hopping events and charge motion is a homogeneous random walk, the diffusion coefficient $D$ could be evaluated from the hopping rate as: 


$$
D=\frac{1}{2 n} \sum_{i} r_{i}^{2} k_{i} P_{i}
$$

where $n$ is the spatial dimensionally (usually $n$ is equal to 3 ), $r_{i}$ is the centroid distance of the hopping channel $i$, and $P_{i}$ is the hopping probability for the specific path $i$, which is calculated as:

$$
P_{i}=k_{i}\left(\sum_{i} k_{i}\right)^{-1}
$$

Here, the drift mobility for charge carrier transport in organic semiconductor, $\mu$, can be evaluated from Einstein relation [20]:

$$
\mu=\frac{e}{k_{B} T} D
$$

In the organic single crystals, the orientation function describing the mobility in a specific conducting direction on a specific surface can be written as the following equation, which is known as the angular resolution anisotropic mobility [21]:

$$
\mu_{\Phi}=\frac{e}{2 k_{B} T} \sum_{i} r_{i}^{2} k_{i} P_{i} \cos ^{2} \gamma_{i} \cos ^{2}\left(\theta_{i}-\Phi\right)
$$

here $\gamma_{i}$ is the angle of hopping path relative to the plane of molecular stacking layer, $\Phi$ is the orientation angle of the transistor channel relative to the specific crystallographic axis and $\theta_{i}$ is the angle of the projected hopping path relative to the specific axis.

\subsection{Computational details}

For molecules ATT, ATT1, ATT2 and ATT3, the optimized geometry has been obtained by DFT calculations at the GGA/PBE level, and Gaussian electrostatic potential (ESP) charges of all atoms were obtained. The crystal structure prediction, based on the embedded ESP charges, was performed by employing the Dreiding force field in the Polymorph Predictor (PP) module of Materials Studio package [6], which is considered to be more appropriate force fields for molecular crystal prediction [22]. The 
van der Waals energy of crystal structure was calculated by the Ewald summation in direct space with a cutoff of $6.0 \AA$ and an accuracy of $10^{-4} \mathrm{kcal} \cdot \mathrm{mol}^{-1}$. For the four ATT molecules, the PP calculations are restricted to the seven most probable space groups, $P 2_{1} / c, P 1, P b c a, P 2_{1} 2_{1} 2_{1}, P 2_{1}, C 2 / c$, and $P-1$, because the statistical result from the Cambridge Structural Database system has illustrated that most of organic compounds belong to these seven space groups [23]. The generated structures for all space groups are arranged according to their energy in the list starting from the structure with the lowest energy. For the ATT, ATT1, ATT2 and ATT3, the predicted crystal cell parameters for the ten crystal structures with the lower total energies are given in Supporting Information, Table S1-S4. Two predicted crystal structures with the lower energies were selected for further DFT calculations by using the dispersion-corrected density functional theory (DFT-D) at the PBE level. Inclusion of dispersion energy (-D) is usually needed to describe the solid-state packing of molecules [24]. The selected optimized crystal cell parameters of the four compounds with the two lower total energies are listed in Table S5. The predicted crystal structure parameters for ATT based on the above method were summarized as: $a=11.94 \AA, b=4.92 \AA, c=14.70 \AA, \alpha=90^{\circ}, \beta=91.45^{\circ}$ and $\gamma=90^{\circ}$. The experimental unit cells of the crystal structure ATT was retrieved from the Cambridge Crystallographic Database ( $a=11.29 \AA, b=5.03 \AA$, $c=14.31 \AA, \alpha=90^{\circ}, \beta=92^{\circ}$ and $\left.\gamma=90^{\circ}\right)$. It is worth noting that the optimized crystal structure parameters are in good agreement with the experiment, demonstrating that the method is suitable for predicting the crystal structures of a series of ATT molecules.

Numerous theoretical works have shown that B3LYP functional is considered to give good evolution on the reorganization energy for the sulphur-bearing aromatic $\pi$-conjugated organic molecules $[25,26]$. So fully geometrical optimizations for ATT, ATT1, ATT2 and ATT3 (shown in Figure 1) in their neutral, cationic and anionic states were performed at the B3LYP/6-31G(d,p) level [27]. Then the 
reorganization energies for the four compounds were calculated using the adiabatic potential approach (AP). All the above calculations were performed with the Gaussian 09 program package [28]. Huang et al. have reported that the PW91PW91 functional gave the best description for the bandwidth of organic solid, and researchers obtained very good results of transfer integral using PW91PW91/6-31G(d,p) level [29]. Herein, the transfer integral calculations for the dimers were evaluated at the PW91PW91/6-31G(d,p) level by the site-energy corrected method using the AOMix package [30].

\section{Results and discussion}

\subsection{Geometrical structure and reorganization energy}

For ATT1, ATT2 and ATT3, the predicted crystal structures with the lowest-energy were used for investigation of the transport properties. The selected optimized geometric parameters, including bond length, bond angles and dihedral angles of ATT, ATT1, ATT2 and ATT3 molecules both neutral and ionic states based on B3LYP/6-31G(d,p) method are collected in Table S6-S9. The absolute variations of bond lengths $(\Delta R)$, bond angles $(\Delta A)$ and dihedral angles $(\Delta D)$ between ionic and neutral states for the four compounds are found to be in the ranges of $0.000-0.019 \AA, 0.041-0.163^{\circ}$ and $0.000-0.046^{\circ}$, respectively. As shown in Figure 2, it can be seen that structure modifications for all the molecules upon oxidation and reduction occur mainly on the bond angles in the peripheral thiophene and the bond lengths in the terminal phenyl ring. It is noted that the difference of dihedral angles between ionic and neutral states for the four molecules are less than $0.1^{\circ}$, indicating that the four molecules exhibit excellent planar structures upon the charge carrier transport process. As can be seen, for the two isomers ATT1 and ATT2, the attachment of fluorine atoms in the diagonal positions of ATT2 slightly shortens the C-S bond by the $0.004 \AA$, and prolongs the $\mathrm{C} 3-\mathrm{C} 4$ bond by $0.009 \AA$ and $\mathrm{C} 4-\mathrm{C} 5$ bond by $0.008 \AA$ compared with ATT. 
When the fluorine substituents are on the same side of ATT1, the change of the bond length is in the range of $0.000-0.003 \AA$. Notably, the changes of bond length S2-C3, bond angle C1-S2-C3 and dihedral angle C1-S2-C3-C4 of ATT2 upon the oxidation are larger than other three molecules, indicating that the fluorine atoms at the diagonal positions incur the obvious geometric changes upon oxidation process. The average variations of dihedral angles from neutral to anionic states decrease in the order of ATT3 $>$ ATT1 $>$ ATT $>$ ATT2. For the ATT1 and ATT3, the dihedral angles between the thiophene and terminal phenyl ring are easy to twist upon the reduction process due to the steric hindrance of fluorines at the homolateral thiophene rings. Overall, the variation in the number and location of fluorine atoms of ATT derivatives will lead to different changes in the geometrical structures.

The reorganization energies are in proportion to the deformation of the geometries in charge transfer process [31]. As seen from Figure 2, for molecules ATT, ATT1 and ATT3, it can be found that their geometrical deformations accompanying electron transfer are larger than the corresponding hole ones, so the three molecules have larger electron reorganization energies $\left(\lambda_{\mathrm{e}}\right)$ compared to their hole reorganization energy $\left(\lambda_{\mathrm{h}}\right)$. However, the molecule ATT2 is more rigid and undergoes smaller geometry relaxations during the electron transfer process compared to other three compounds, which indicates that the molecule ATT2 may has the smallest electron reorganization energy. The $\lambda_{\mathrm{h}}$ and $\lambda_{\mathrm{e}}$ for the ATT derivatives were calculated by adiabatic potential surfaces (AP) method at the B3LYP/6-31G(d,p) level and the corresponding values are collected in Table 1 [16]. The magnitude of $\lambda_{\mathrm{e}}$ of ATT, ATT1 and ATT3 is somewhat larger than its corresponding $\lambda_{\mathrm{h}}$. The $\lambda_{\mathrm{h}}$ decrease in the order of ATT2 $>$ ATT3 $>$ ATT1 $>$ ATT, while the $\lambda_{\mathrm{e}}$ follow the order of ATT3 $>$ ATT1 $>$ ATT $>$ ATT2, which agrees well with the geometrical deformations upon charge transfer process analysis discussed above. The different evolutionary trends of $\lambda_{\mathrm{h}}$ and $\lambda_{\mathrm{e}}$ could be explained in terms of the geometry changing when the 
electronic states changes. In comparison with ATT1, ATT2 is more suitable for electron transport semiconductor from the viewpoint of reorganization energy. For the ATT3, there is no doubt that the more electron-withdrawing atoms will unavoidably induce more vibrational modes of geometric relaxation [11], which will contribute to an increased electron reorganization energy. Generally, the calculated $\lambda_{\mathrm{e}}$ values for the four compounds $(0.149-0.177 \mathrm{eV})$ are lower than the typical n-type organic semiconductor perfluoropentacene $(0.24 \mathrm{eV})$ [32]. This demonstrates that the replacement of hydrogen by fluorine at some positions is beneficial to electron transport.

\subsection{Frontier molecular orbital, ionization potential (IP) and electron affinity (EA)}

The energies of the lowest unoccupied molecular orbitals (LUMOs) and the highest occupied molecular orbitals (HOMOs) provide a reasonable qualitative indication for the electron and hole injection, respectively [33]. The calculated HOMOs and LUMOs as well as the HOMO-LUMO gaps for the four compounds are depicted in Figure 3. The attachment of fluorine substituents to the conjugated thiophene could decrease both HOMO and LUMO levels to an extent and the LUMO levels are much more lowered. The HOMO levels of ATT1, ATT2 and ATT3 are $-5.349,-5.341$ and $-5.462 \mathrm{eV}$, respectively, which are consistent well with the high work-function metal of gold $(5.1 \mathrm{eV})$ [34]. The lowered LUMO levels facilitate the injection of electrons effectively which is necessary for an n-type organic semiconductor and which also contributes to its stability in the environment. Obviously, the ATT3 has the lowest low-lying LUMOs energy, indicating that electron is easier to be injected into the ATT3. It is noted that with increasing number of substituent fluorine atoms, the HOMO-LUMO gap of the ATT derivatives continuously decreases. The smaller the barrier is, the easier the charge injection is. Apparently, the HOMO-LUMO gap of ATT3 is smaller than those of other compounds, which is 
beneficial to electron transport. For ATT1 and ATT2, it can be found that the position of the fluorine atoms strongly influences their frontier orbitals. The contribution of fluorine atoms to its LUMO and HOMO for ATT2 is larger than that for ATT1. The former possesses lower LUMO level and higher HOMO level, and hence better electron injecting ability.

To better understand the role of fluorine atoms in their HOMOs and LUMOs, the corresponding electronic density contours of all the investigated compounds are displayed in Figure 4. All the frontier molecular orbitals possess $\pi$ orbital features, which is dominantly due to the $p_{z}$ orbital of the carbon atoms [35]. The HOMO of the title compounds is delocalized over the entire molecule with large density on the C-C bonds of the center anthracene and the C-S bonds of the thiophene rings. The LUMO of four compounds is located over the skeleton anthra-tetrathiophene region of the molecule especially the carbon and sulfur atoms. When introducing electron-withdrawing fluorine atoms to the ATT, both the HOMO and LUMO are delocalized on the substituted fluorine atoms. Thus, it can be speculated that the decrease of HOMO and LUMO energies of ATT derivatives is mainly attributed to the $\pi$-conjugation extension to fluorinated thiophene ring. This distribution nature of HOMO and LUMO is expected to favor the charge transfer along the $\pi$-orbital of nearby molecules.

Ionization potentials $(I P)$ and electron affinities $(E A)$ of all studied compounds were calculated at the B3LYP/6-31G(d,p) by the DFT method and the results are listed in Table 2. The IP and EA are generally used to characterize the reduction and oxidation ability, which can also provide useful information regarding the organic device performance and its ambient stability [36]. The ionization potential relates to the holes injection to HOMO and low-ionization potentials are desired for the p-type materials. Whereas for n-type semiconductor, the electron affinity is expected to be high enough to ensure that the electrons come across the energy barrier and allow efficient injection of the electrons into 
the empty LUMO [37]. The small difference in the vertical and adiabatic values indicates that the structural relaxation upon charge injection is small. It can be found that these molecules with fluorine atoms possess large $E A_{V}(0.759-0.927 \mathrm{eV})$ and $I P_{V}(6.588-6.671 \mathrm{eV})$ values due to their low LUMO and HOMO levels. Their large $E A_{V}$ values give rise to high stability of their radical anions in ambient atmosphere, which is an important requirement of n-type OFET materials. In particular, the electron affinities of the derivatives are stabilized when their $E A_{V}$ values are $0.8-1.8 \mathrm{eV}$ [16]. With the largest $E A_{V}$ being up to $0.927 \mathrm{eV}$, the ATT3 should be stable in the ambient environment. According to Table 2, both $I P$ and $E A$ have been increased obviously after the attachment of fluorine atoms. The enhanced electron affinity facilitates the electrons injecting into LUMOs of semiconductors effectively.

\subsection{Transfer integrals, packing motif and intermolecular interaction}

The charge transport properties of conjugated materials critically depend on the transfer integrals, which are very sensitive to the relative position of the adjacent molecules and their packing manner in the crystal [38]. Based on the crystal structures, the main charge transfer pathways along the long and short axis of the ATT, ATT1, ATT2 and ATT3 are depicted in Figure 5. It is worth to notice that the four compounds have rigorous planar structure and mainly employ the face-to-edge and face-to-face $(\pi-\pi)$ stacking. Obviously, the packings of dimers P1 and P2 of the four compounds adopt the slipped $\pi-\pi$ stacking, which is a favorable packing with small energetic disorder and beneficial topology of charge percolating network, while the arrangement of other dimers are completely shifted with face-to-edge packing [39]. The transfer integral of each pathway was calculated at the PW91PW91/6-31G(d,p) level by the site-energy corrected method and the corresponding values were listed in Table 3 [28]. Besides, the $J, S$ and $E$ values for the hole and electron transport of the ATT and its three fluorinated derivatives 
were collected in Table S10-S13. As shown in Table 3, since both the calculated reorganization energies of hole and electron transport for the ATT derivatives are larger than the largest hole and electron transfer integral, the localized description of the charge transfer by the Marcus-Hush model is adequate for investigating the charge mobilities of the four compounds [40]. The transfer integral in various hopping pathways are remarkably different. The $\pi-\pi$ stacking of the dimers P1 and P2 with the minimal interplane distances have the largest transfer integral values, which demonstrates that the charge carrier mobility of each crystal is mainly determined by one dimensional charge transport. This finding further proved the traditional view that the face-to-face stacking with a short distance and the large coupling area could contribute to the larger electron coupling. In comparison with ATT, the introduction of two fluorine substituents increases the hole and electron transfer integral for ATT2, while decreases the electron transfer integral for ATT1 in main hopping pathways, which indicates that the introduction of fluorine atoms at the diagonal directions may be beneficial to electron transport from the viewpoint of transfer integral. By attaching four fluorine atoms to the thiophene rings, the electron transfer integral increases profoundly, and ATT3 has the largest electron transfer integral due to its larger orbital overlap between adjacent molecules, which indicates that the more electron-withdrawing fluorine substituents could largely improve electron transport.

The transfer integral also depends on the overlap degree and phase of the interacting molecular orbital [41]. To further understand the intermolecular effective electronic couplings, the overlap of HOMOs and LUMOs orbitals of the dimer P1 with the maximal transfer integral for the four compounds are presented in Figure 6 . When the $\pi$ orbitals between the neighbor molecules involve the bonding or antibonding interactions, the transfer integral of the dimer will increase remarkably [42]. As shown in Figure 6, the wave functions of the interacting monomers for ATT1 and ATT3 at the overlapping region 
possess the same phase. ATT1 has largest hole transfer integral $(-127.43 \mathrm{meV})$ and ATT3 has the largest electron transfer $(-83.81 \mathrm{meV})$ since the dimers P1 exhibit optimal $\pi$-orbital overlap upon the hole transfer process for ATT1 and the electron transfer process for ATT3, respectively. A close examination reveals that the strong inter orbital overlaps results from the $\pi$ - $\pi$ interactions between the neighboring molecules. However, the dimer P1 for ATT1 possesses the minimal electron transfer integral because a cancellation between bonding and antibonding overlaps weakens the electronic coupling between the interacting molecules. It is noted that for the four compounds, the vertical distance of dimer P1 between the neighboring molecules is quite small, while the mass center distances of other dimers are larger, especially dimers P9 and P10. Generally, the value of transfer integral depends on the distance between the intermolecular monomers directly interacting with each other. When the mass center distance of the dimer is too far, the transfer integral is close to zero. Herein, the values of transfer integral of the dimers P9 and P10 are very small, which are almost negligible compared with other dimers. Overall, the larger overlap area of the $\pi$-orbital and the smaller vertical distance between neighboring molecules contribute to the larger transfer integrals.

\subsection{Charge carrier mobility and its anisotropy}

Based on the calculated reorganization energy and transfer integral, the carrier mobility for both hole and electron are evaluated at 300K using the Equations 1, 5 and 6, and the corresponding results are given in Table 4 . The hole mobility $\left(\mu_{\mathrm{h}}\right)$ of ATT is $1.16 \mathrm{~cm}^{2} \cdot \mathrm{V}^{-1} \cdot \mathrm{s}^{-1}$, larger than the experimental value of $0.012 \mathrm{~cm}^{2} \cdot \mathrm{V}^{-1} \cdot \mathrm{s}^{-1}$. The discrepancy between the theoretical and experiment value is accepted, which may originate from some possible reasons, such as the influence of carrier trap, energy traps and grain boundary in experiments, the approximation of the Equation 1 for hopping in theory $[43,44]$. It can be 
found that the position of fluorine atoms in the thiophene ring could change electron mobilities. The electron mobility of ATT2 $\left(0.41 \mathrm{~cm}^{2} \cdot \mathrm{V}^{-1} \cdot \mathrm{s}^{-1}\right)$ with the two fluorine atoms at the diagonal directions is much larger than its isomer ATT1 $\left(0.02 \mathrm{~cm}^{2} \cdot \mathrm{V}^{-1} \cdot \mathrm{s}^{-1}\right)$ due to the smaller electron reorganization energy and larger electron transfer integrals of the former. Besides, the $\mu_{\mathrm{h}}$ of the ATT2 $\left(0.85 \mathrm{~cm}^{2} \cdot \mathrm{V}^{-1} \cdot \mathrm{s}^{-1}\right)$ is slightly larger than its $\mu_{\mathrm{e}}$. The same order of magnitude for hole and electron transfers suggests that the ATT2 crystal should be a potential ambipolar organic semiconductor material. When four hydrogen atoms are replaced by fluorine atoms, ATT3 possesses the largest electron mobility $\left(0.48 \mathrm{~cm}^{2} \cdot \mathrm{V}^{-1} \cdot \mathrm{s}^{-1}\right)$ due to large and direct $\pi-\pi$ overlap, in despite of its large electron reorganization. Obviously, the $\mu_{\mathrm{e}}$ of ATT3 is quite larger than its corresponding $\mu_{\mathrm{h}}\left(0.08 \mathrm{~cm}^{2} \cdot \mathrm{V}^{-1} \cdot \mathrm{s}^{-1}\right)$ in that the electronic coupling between LUMOs has been enhanced significantly, about three orders of magnitude larger than that of HOMOs, which demonstrates that ATT3 is more suitable for an n-type organic semiconductor. Saranya et al. had reported the $\mathrm{NO}_{2}$ substituted ATT molecule is a high-performance n-type organic semiconductor and its electron mobility reaches up to $0.31 \mathrm{~cm}^{2} \cdot \mathrm{V}^{-1} \cdot \mathrm{s}^{-1}$ [35]. When two/four hydrogen atoms are replaced by fluorine atoms of the ATT molecule, the electron mobilities of ATT2 and ATT3 are 0.41 and 0.48 $\mathrm{cm}^{2} \cdot \mathrm{V}^{-1} \cdot \mathrm{s}^{-1}$, respectively, larger than the electron mobility of the $\mathrm{NO}_{2}$ substituted ATT molecule. Besides, the $\mu_{\mathrm{e}}$ of ATT2 and ATT3 are more than $0.1 \mathrm{~cm}^{2} \cdot \mathrm{V}^{-1} \cdot \mathrm{s}^{-1}$, the threshold value fully meeting the practical OFET application [45], which further indicates that ATT2 and ATT3 can be function as high-performance ambioplar or n-type organic semiconductor. Overall, an introduction of the suitable number of electron-withdrawing fluorine atoms to the $\pi$-conjugated molecules not only guarantees the fluorinated molecular coplanarity, but also enhances $\pi-\pi$ overlap area, which will improve the electron transport.

As we all known, the anisotropy is originated from the variations of the intermolecular electronic 
coupling strength, center-to-center distance and molecular packing modes. According to the values of the transfer integral in Table 3, we have speculated the charge carrier mobilities of the four compounds are remarkably anisotropic $[21,46]$. Understanding the anisotropic mobility can assist in controlling the directions of transistor channel relative to reference direction of molecular crystal to obtain the highest charge mobility. Here, taking the excellent extended $\pi$-conjugated system ATT2 and ATT3 as examples, the angular resolution electron mobility has been discussed through the $b-c$ plane. The angular resolution anisotropic hole and electron mobilities in ATT2 and ATT3 crystals are depicted in Figure 7 and Figure 8, respectively. As seen from Figure 7 and Figure 8, the distributions of their mobilities vary a lot along the different directions and express the representative angular resolution anisotropic characteristic. The hole mobility of ATT2 is obviously larger than its electron mobility since the strongest coupling of HOMOs occurs in the crystal ATT2. Notably, at the reference angle of $0^{\circ}$ and $180^{\circ}$, maxima of hole $(1.27$ $\left.\mathrm{cm}^{2} \cdot \mathrm{V}^{-1} \cdot \mathrm{s}^{-1}\right)$ and electron $\left(0.62 \mathrm{~cm}^{2} \cdot \mathrm{V}^{-1} \cdot \mathrm{s}^{-1}\right)$ mobilities for ATT2 can be found, while the minima appear in the $c$-axis direction, which correspond to the reference angle of close to $90^{\circ}$ and $270^{\circ}$. Similarly, as for ATT3, the highest hole $\left(0.11 \mathrm{~cm}^{2} \cdot \mathrm{V}^{-1} \cdot \mathrm{s}^{-1}\right)$ and electron $\left(0.72 \mathrm{~cm}^{2} \cdot \mathrm{V}^{-1} \cdot \mathrm{s}^{-1}\right)$ mobilities are along the $0^{\circ}$ and $180^{\circ}$ directions relative to the $b$-axis direction, while its lowest hole and electron mobilities are also perpendicular to the axes in the $b-c$ plane. Interestingly, the maximum electron mobility value is nearly 7 times larger than its corresponding maximum hole mobility for ATT3, which indicates that electrons are intrinsically far more mobile than holes in the ATT3 crystal. Additionally, the largest electron mobility in the special direction for ATT3 crystal probably makes it widely applicable as an n-type organic semiconductor. It also can be found that the directions of the largest hole and electron mobilities for ATT2 and ATT3 correspond to the transport pathway with $\pi-\pi$ interaction possessing larger transfer integrals (along the $\mathrm{P}$ direction). These results further suggest that the $\pi-\pi$ interactions are the main 
driving force to form such anisotropic crystals, and fortunately it is helpful for carrier transport because the $\pi-\pi$ stacking direction is usually a large carrier transporting direction.

\subsection{Band structure}

To gain a further understanding of the anisotropy of charge carrier transport in single crystals, the band structures of ATT, ATT1, ATT2 and ATT3 were also investigated and shown in Figure 9. The band gaps of the ATT, ATT1, ATT2 and ATT3 are 1.983, 1.837, 1.827 and $1.785 \mathrm{eV}$, respectively. Generally, the appearances of bands, dispersive or flat, are reflections of the anisotropy in the charge transports of the crystal. A stronger dispersion of the band indicates that the crystal possesses larger carrier mobility [47]. It can be seen that both valence bands (VBs) and conduction bands (CBs) consist of two sub-bands on account of the two irreducible molecules in unit cell. As shown in Figure 9, ATT1 has the strongest dispersion of VB and ATT3 possesses the strongest dispersion of CB among the four crystals, therefore ATT1 has the largest hole mobility and ATT3 has the largest electron mobility. For the ATT, the strongest valence band dispersion occurs in the GY subzone, corresponding to the $b$ axis in real space, and the dimers P1 and P2 with the maximal hole transfer integral are along this direction. Whereas, for the ATT3, by introducing four fluorine atoms to the thiophene, the largest conduction band dispersions only along the GY direction correspond to the $\pi-\pi$ packing direction with large transfer integrals, indicating that the ATT3 present 1-D electron transport behavior. As for ATT1, the flat conduction band in all directions suggests that its electron mobility is very low compared with other crystals. It is interested to find that the bandwidth in VB is much larger than that in $\mathrm{CB}$, indicating that the hole mobility of ATT1 is larger than that of electron mobility. However, the largest dispersion in CB is broader than that in VB of ATT3, suggesting that the electron transfer ability is superior to the hole one, 
which demonstrates that ATT3 can act as an electron transport material, which is well consistent with the calculated hole and electron mobilities above. For the ATT2, the bandwidth of CB is close to the VB, which indicates that the ATT2 is a promising ambipolar organic material.

\section{Conclusion}

The combination of the Monte Carlo-simulated annealing method with DFT-D is adequate to predict the crystal structure of ATT and its fluorinated derivatives. The charge carrier transport properties of the title compounds have been investigated by DFT techniques and Marcus-Hush theory. The molecular geometry, reorganization energy, frontier orbitals, IPs and EAs, transfer integrals, charge mobility as well as band structure have been explored. With the number of fluorine atoms increasing from ATT to ATT3, the HOMO and LUMO become more delocalized and the HOMO-LUMO gap more narrowed which is beneficial to electron injection and provides more charge carrier stabilization. Attaching fluorine to ATT could affect crystal packing and hence the transfer integral. The larger transfer integral depends mainly on the face-to-face $\pi-\pi$ stacking, which is considered to be a favorable packing and beneficial topology of charge percolating network. Meantime, the $\pi-\pi$ stacking with a short distance and the large coupling area could contribute to the largest electron coupling. Among the four compounds, although ATT3 has the largest electron reorganization energy, it has the largest electron mobility $(0.48$ $\mathrm{cm}^{2} \cdot \mathrm{V}^{-1} \cdot \mathrm{s}^{-1}$ ) due to large and direct $\pi-\pi$ overlap and the LUMO levels. ATT2 and ATT3 single crystals display remarkable anisotropic charge transport behavior. The band structures suggested that the paths with large transfer integrals are all along the directions with large dispersions in VB and CB. The number and location of electron-withdrawing fluorine atoms could significantly affect the electron transport and provide a new strategy for the design of high-performance n-type organic semiconductor. 


\section{Acknowledgements}

The authors thank the National Science Foundation of China (No. 21372116) as well as the Projects Funded by China Postdoctoral Science Foundation (No. 2015M581083) and by the Priority Academic Program Development of Jiangsu Higher Education Institutions (PAPD) for supporting this work.

\section{References}

[1] Y. Ie, T. Uto, N. Yamamoto, Y. Aso, Dendritic oligothiophene bearing perylene bis(dicarboximide) groups as an active material for photovoltaic device, Chem. Commun. 23 (2009) 1213-1215.

[2] M. L. Tang, T. Okamoto, Z. N. Bao, High-performance organic semiconductors: asymmetric linear acenes containing sulphur, J. Am. Chem. Soc. 128 (2006) 16002-16003.

[3] L. L. Chua, J. Zaumseil, J. F. Chang, H. Sirringhaus, R. H. Friend, General observation of n-type field-effect behaviour in organic semiconductors, Nature 434 (2005) 194-199.

[4] L. Jiang, W. P. Hu, Z. M. Wei, W. Xu, H. Meng, High-performance organic single-crystal transistors and digital inverters of an anthracene derivative, Adv. Mater. 21 (2009) 3649-3653.

[5] P. F. Baude, D. A. Ender, M. A. Haase, T. W. Kelley, D. V. Muyres, S. D. Theiss, Pentacene-based radio-frequency identification circuitry, Appl. Phys. Lett. 82 (2003) 3964-3966.

[6] W. J. Liu, Y. Zhou, Y. Ma, Y. Cao, J. Wang, J. Pei, Thin film organic transistors from air-stable heteroarenes: anthra-[1,2-b:4,3-b':5,6-b":8,7-b"']tetrathiophene derivatives, Org. Lett. 9 (2007) $4187-4190$.

[7] W. P. Wu, Y. Q. Liu, D. B. Zhu, $\pi$-Conjugated molecules with fused rings for organic field-effect transistors: design, synthesis and applications, Chem. Soc. Rev. 39 (2010) 1489-1502.

[8] J. L. Brusso, O. D. Hirst, A. Dadvand, S. Ganesan, F. Cicoira, C. M. Robertson, R. T. Oakley, F. 
Rosei, D. F. Perepichka, Two-dimensional structural Motif in thienoacene semiconductors: synthesis, structure, and properties of tetrathienoanthracene isomers, Chem. Mater. 20 (2008) $2484-2494$.

[9] C. Röger, F. Würthner, Core-Tetrasubstituted Naphthalene Diimides: Synthesis, Optical Properties, and Redox Characteristics, J. Org. Chem. 72 (2007) 8070-8075.

[10] J. C. Sancho-García, A. J. Pérez-Jiménez, Y. Olivier, J. Cornil, Molecular packing and charge transport parameters in crystalline organic semiconductors from first-principles calculations, Phys. Chem. Chem. Phys. 12 (2010) 9381-9388.

[11] S. Chai, S. H. Wen, J. D. Huang, K. L. Han, Density functional theory study on electron and hole transport properties of organic pentacene derivatives with electron-withdrawing substituent, J. Comput. Chem. 2011 3218-3224.

[12] Z. G. Shuai, L. Wang, Q. Li, Evaluation of charge mobility in organic materials: from localized to delocalized descriptions at a first-principles level, Adv. Mater. 23 (2011) 1145-1153.

[13] J. Cornil, J. L. Brédas, J. Zaumseil, H. Sirringhaus, Ambipolar transport in organic conjugated materials, Adv. Mater. 19 (2007) 1791-1799.

[14] B. S. Brunschwig, J. Logan, M. D Newton, N. Sutin, A semiclassical treatment of electron-exchange reactions. application to the hexaaquoiron(II)-hexaaquoiron(III) system, J. Am. Chem. Soc. 102 (1980) 5798-5809.

[15] M. D. Newton, N. Sutin, Electron transfer reactions in condensed phases. Annu. Rev. Phys. Chem. 35 (1984) 437-480.

[16] M. Malagoli, J. L. Brédas, Density functional theory study of the geometric structure and energetics of triphenylamine-based hole-transporting molecules, Chem. Phys. Lett. 327 (2002) 13-17. 
[17] L. J. Wang, P. Li, B. Xu, H. Y. Zhang, W. J. Tian, The substituent effect on charge transport property of triisopropylsilylethynyl anthracene derivatives, Org. Electron. 15 (2014) 2476-2485.

[18] E. F. Valeev, V. Coropceanu, D. A. da Silva Filho, S. Salman, J. L. Brédas, Effect of electronic polarization on charge-transport parameters in molecular organic semiconductors, J. Am. Chem. Soc. 128 (2006) 9882-9886.

[19] P. Lwdin, On the non-orthogonality problem connected with the use of atomic wave functions in the theory of molecules and crystals, J. Chem. Phys. 18 (1950) 365-375.

[20] J. Cornil, V. Lemaur, J. P. Calbert, J. L. Brédas, Charge transport in discotic liquid crystals: a molecular scale description, Adv. Mater. 14 (2002) 726-729.

[21] S. H. Wen, A. Lin, J. L. Song, W. Q. Deng, K. L. Han, W. A. Goddard III. First-principles investigation of anistropic hole mobilities in organic semiconductors, J. Phys. Chem. B 113 (2009) $8813-8819$.

[22] S. L. Mayo, B. D. Olafson, W. A. Goddard III, DREIDING: a generic force field for molecular simulations, J. Phys. Chem. 94 (1990) 8897-8909.

[23] Y. F. Cheng, Z. Y. Lu, L. J. An, J. P. Zhang, From molecules to materials: molecular and crystal engineering design of organic optoelectronic functional materials for high carrier mobility, J. Phys. Chem. C 116 (2012) 1195-1199.

[24] J. P. Perdew, K. Burke, M. Ernzerhof, Generalized gradient approximation made simple, Phys. Rev. Lett. 77 (1996) 3865-3868.

[25] M. C. R. Delgado, E. G. Kim, D. A. daSilvaFilho, J. L. Brédas, Tuning the charge-transport parameters of perylene diimide single crystals via end and/or core functionalization: a density functional theory investigation, J. Am. Chem. Soc. 132 (2010) 3375-3387. 
[26] P. Song, F. C. Ma, Tunable electronic structures and optical properties of fluorenone-based molecular materials by heteroatoms, J. Phys. Chem. A 114 (2010) 2230-2234

[27] C. H. Li, C. H. Huang, M. Y. Kuo, Halogenated 6,13-bis(triisopropylsilylethynyl)-5,7,12,14-tetraazapentacene: applications for ambipolar air-stable organic field-effect transistors, Phys. Chem. Chem. Phys. 13 (2011) 11148-11155.

[28] M. J. Frisch, G. W. Trucks, H. B. Schlegel, G. E. Scuseria, M. A. Robb, J. R. Cheeseman, G. Scalmani, V. Barone, B. Mennucci, G. A. Petersson, H. Nakatsuji, M. Caricato, X. Li, H. P. Hratchian, A. F. Izmaylov, J. Bloino, G. Zheng, J. L. Sonnenberg, M. Hada, M. Ehara, K. Toyota, R. Fukuda, J. Hasegawa, M. Ishida, T. Nakajima, Y. Honda, O. Kitao, H. Nakai, T. Vreven, J. A. Montgomery, Jr., J. E. Peralta, F. Ogliaro, M. Bearpark, J. J. Heyd, E. Brothers, K. N. Kudin, V. N. Staroverov, R. Kobayashi, J. Normand, K. Raghavachari, A. Rendell, J. C. Burant, S. S. Iyengar, J. Tomasi, M. Cossi, N. Rega, J. M. Millam, M. Klene, J. E. Knox, J. B. Cross, V. Bakken, C. Adamo, J. Jaramillo, R. Gomperts, R. E. Stratmann, O. Yazyev, A. J. Austin, R. Cammi, C. Pomelli, J. W. Ochterski, R. L. Martin, K. Morokuma, V. G. Zakrzewski, G. A. Voth, P. Salvador, J. J. Dannenberg, S. Dapprich, A. D. Daniels, O. Farkas, J. B. Foresman, J. V. Ortiz, J. Cioslowski, and D. J. Fox, Gaussian 09, Revision A.02, Gaussian, Inc., Wallingford CT, 2009.

[29] J. S. Huang, M. Kertesz, Modeling disorder in polymer aggregates: The optical spectroscopy of regioregular poly(3-hexylthiophene) thin films", J. Chem. Phys. 122 (2005) 234701.

[30] S. I. Gorelsky, S. Ghosh, E. I. Solomon, Mechanism of $\mathrm{N}_{2} \mathrm{O}$ reduction by the $\mu 4-\mathrm{S}$ tetranuclear $\mathrm{Cu}_{\mathrm{Z}}$ cluster of nitrous oxide reductase, J. Am. Chem. Soc. 128 (2006) 278-290.

[31] X. K. Chen, J. F. Guo, L. Y. Zou, A. M. Ren, J. X. Fan, A promising approach to obtain excellent n-type organic field-effect transistors: introducing pyrazine ring, J. Phys. Chem. C 115 (2011) 
$21416-21428$.

[32] Y. Sakamoto, T. Suzuki, M. Kobayashi, Y. Gao, Y. Fukai, Y. Inoue, F. Sato, S. Tokito, Perfluoropentacene: High-performance $\mathrm{p}-\mathrm{n}$ junctions and complementary circuits with pentacene, J. Am. Chem. Soc. 126 (2004) 8138-8140.

[33] A. P. Kulkarni, C. J. Tonzola, A. Babel, S. A. Jenekhe, Electron transport materials for organic light-emitting diodes, Chem. Mater. 16 (2004) 4556-4573.

[34] H. X. Li, R. H. Zheng, Q. Shi, Theoretical study on charge carrier mobilities of tetrathiafulvalene derivatives, Phys. Chem. Chem. Phys. 13 (2011) 5642-5650.

[35] G. Saranya, K. Navamani, K. Senthilkumar, A theoretical study on optical and charge transport properties of anthra-[1,2-b:4,3-b':5,6-b":8,7-b"']tetrathiophene molecules, Chem. Phys. 433 (2014) $48-59$

[36] X. Y. Zhang, G. J. Zhao, J. D. Huang, W. P. Zhang, Effects of carbon chain on hole-transport properties innaphtho[2,1-b:6,5-b']difuran derivatives: remarkable anisotropic mobilities, Org. Electron. 15 (2014) 3341-3348.

[37] Y. C. Chang, M. Y. Kuo, C. P. Chen, H. F. Lu, I. Chao, On the air stability of nchannel organic field-effect transistors: a theoretical study of adiabatic electron affinities of organic semiconductors, J. Phys. Chem. C 114 (2010) 11595-11601.

[38] X. D. Tang, Y. Liao, H. Z. Gao, Y. Geng, Z. M. Su, Theoretical study of the bridging effect on the charge carrier transport properties of cyclooctatetrathiophene and its derivatives, J. Mater. Chem. $22(2012)$ 6907-6918.

[39] M. Schrader, R. Fitzner, M. Hein, C. Elschner, B. Baumeier, K. Leo, M. Riede, P. Bäuerle, D. Andrienko, Comparative study of microscopic charge dynamics in crystalline acceptor-substituted 
oligothiophenes, J. Am. Chem. Soc. 134 (2012) 6052-6056.

[40] L. L. Lin, H. Geng, Z. G. Shuai, Y. Luo, Theoretical insights into the charge transport in perylene diimides based n-type organic semiconductors. Org. Electron. 13 (2012) 2763-2772.

[41] Y. Geng, J. Wang, S. Wu, H. Li, F. Yu, G. Yang, H. Gao, Z. M. Su, Theoretical discussions on electron transport properties of perylene bisimide derivatives with different molecular packings and intermolecular interactions, J. Mater. Chem. 21 (2011) 134-143.

[42] S. F. Zhang, X. K. Chen, J. X. Fan, A. M. Ren, Charge transport properties in a series of five-ring-fused thienoacenes: a quantum chemistry and molecular mechanic study, Org. Electron. 14 (2013) 607-620.

[43] G. Nan, L. Wang, X. Yang, Z. Shuai, Y. Zhao, Charge transfer rates in organic semiconductors beyond first-order perturbation: from weak to strong coupling regimes, J. Chem. Phys. 130 (2009) 024704 .

[44] L. Liu, G. C. Yang, X. D. Tang, Y. Geng, Y. Wu, Z. M. Su, The effect of intermolecular interactions on the charge transport properties of thiazole/thiophene-based oligomers with trifluoromethylphenyl, J. Mol. Graph. Model. 51 (2014) 79-85.

[45] L. Wang, Li Q, Z. G. Shuai, L. Chen, Q. Shi, Multiscale study of charge mobility of organic semiconductor with dynamic disorders. Phys. Chem. Chem. Phys. 12 (2010) 3309-3314.

[46] J. D. Huang, S. H. Wen, W. Q. Deng, K. L. Han, Simulation of hole mobility in $\alpha$-oligofuran crystals, J. Phys. Chem. B 115 (2011) 2140-2147.

[47] Y. A. Duan, H. B. Li, Y. Geng, Y. Wu, G. Y. Wang, Z. M. Su, Theoretical studies on the hole transport property of tetrathienoarene derivatives: the influence of the position of sulfur atom, substituent and p-conjugated core, Org. Electron. 14 (2014) 602-613. 


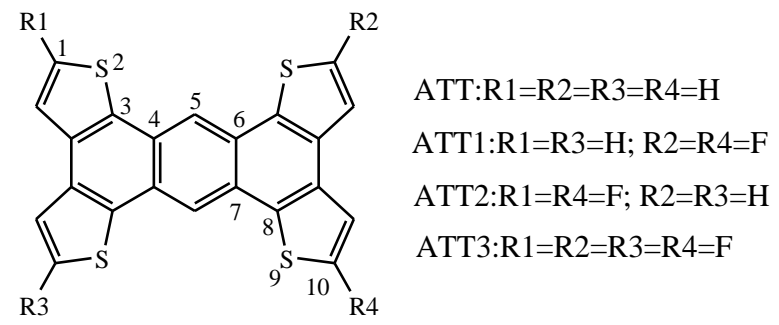

Figure 1. Molecular structures of systems ATT, ATT1, ATT2 and ATT3. 


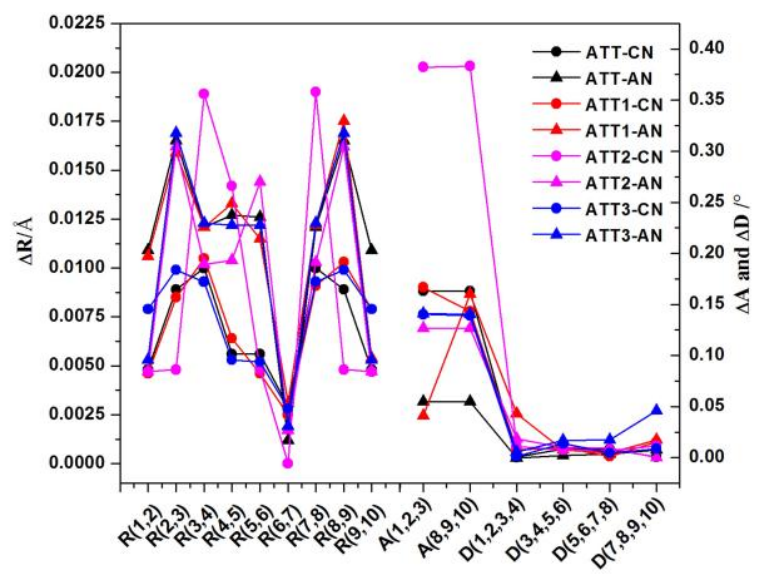

Figure 2. The absolute variations of bond lengths $(\Delta R)$, bond angles $(\Delta A)$, and dihedral angles $(\Delta D)$ between ionic and neutral states in ATT, ATT1, ATT2 and ATT3. CN: the absolute variation between cationic and neutral states. AN: the absolute variation between anionic and neutral states. 


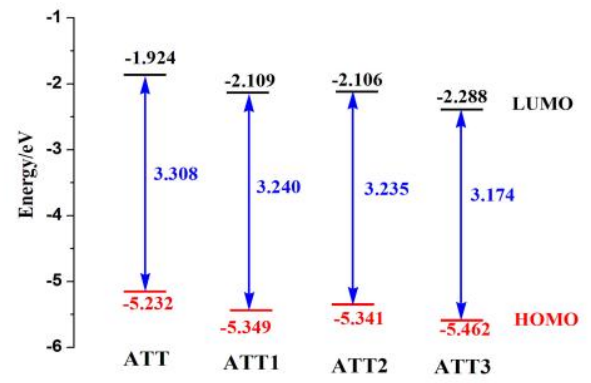

Figure 3. Energy levels (in eV) of HOMO and LUMO of ATT derivatives (the upper ones are LUMOs and the lower ones are HOMOs) with the method B3LYP/6-31G(d,p). 


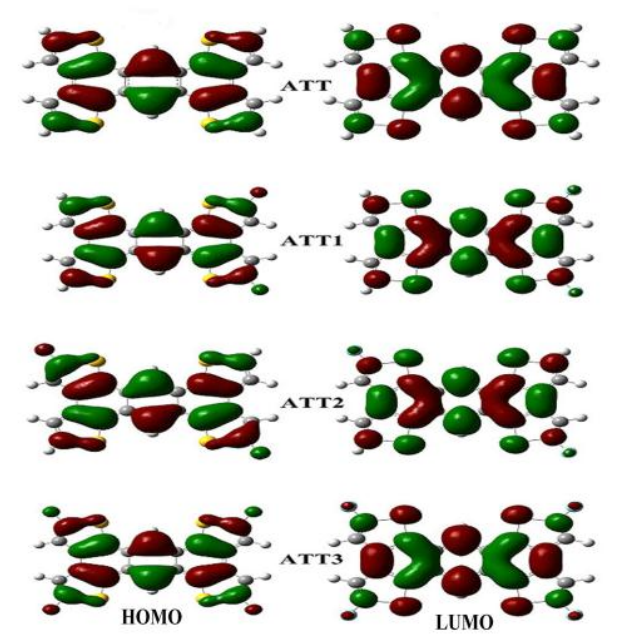

Figure 4. Illustration of distribution of HOMOs and LUMOs of ATT derivatives at the B3LYP/6-31G(d,p) level. 

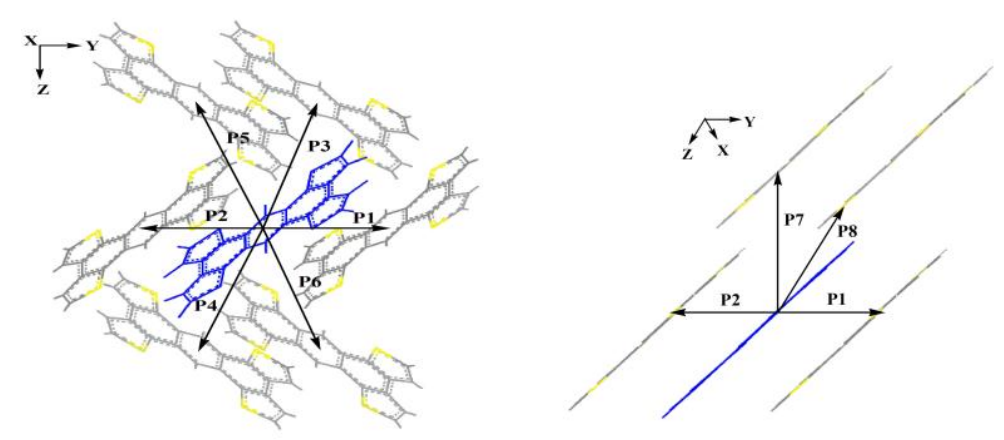

ATT
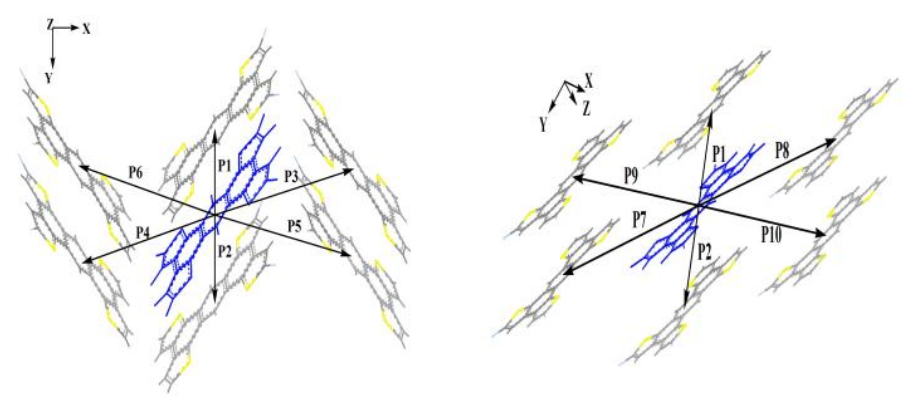

ATT1
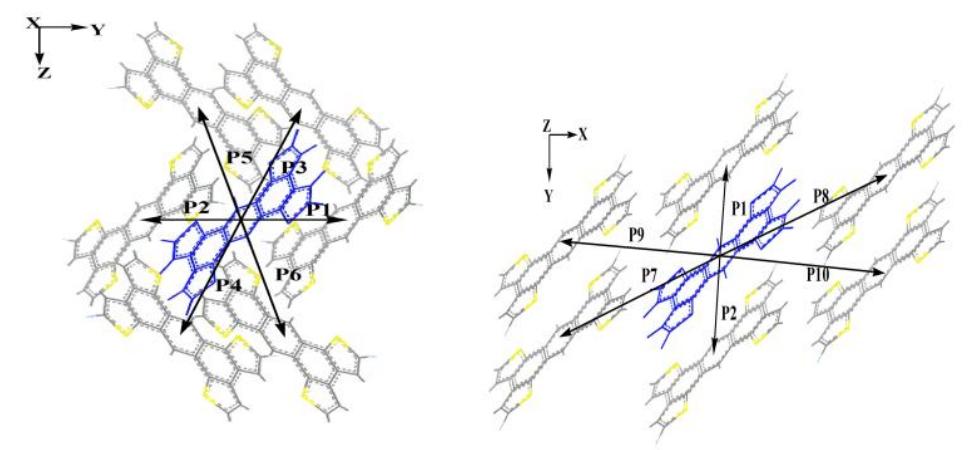

ATT2
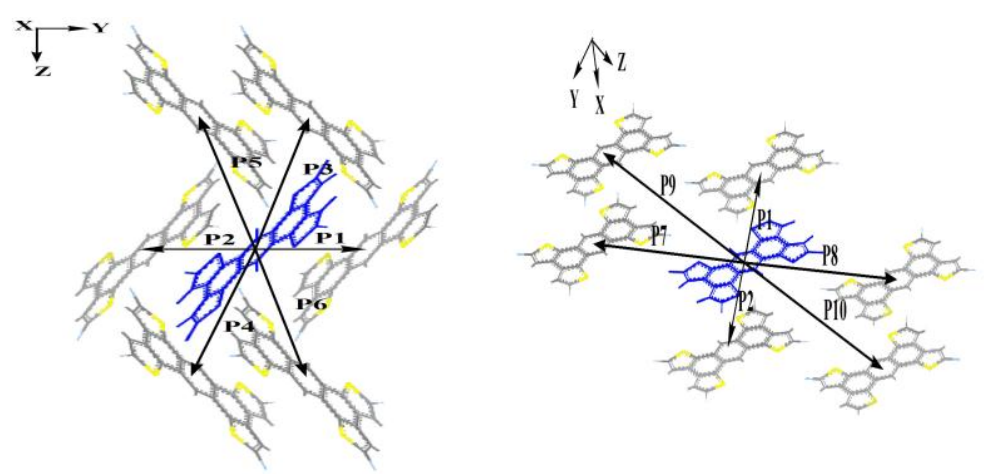

ATT3

Figure 5. Main carrier hopping pathways selected based on the crystals of ATT, ATT1, ATT2 and ATT3. 


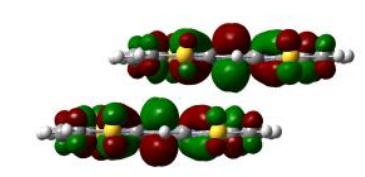

$V_{h}=-70.51$

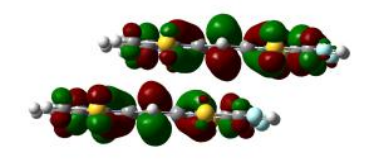

$V_{h}=-127.43$

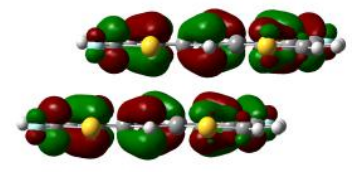

$V_{h}=-96.31$

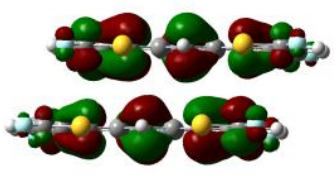

$V_{h}=-30.30$
ATT
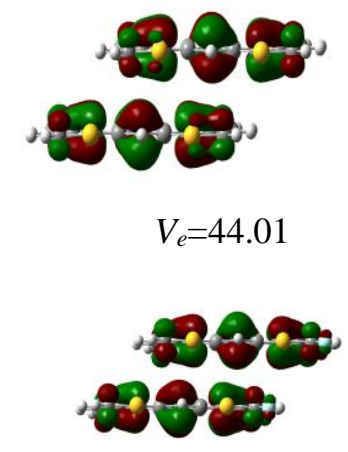

ATT1

ATT2

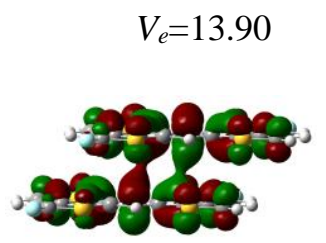

$V_{e}=60.00$

ATT3

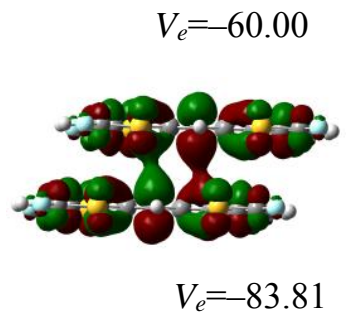

Figure 6. HOMOs (left) and LUMOs (right) interaction as well as the value of transfer integral (in meV) of dimer P1for molecules ATT, ATT1, ATT2 and ATT3. 


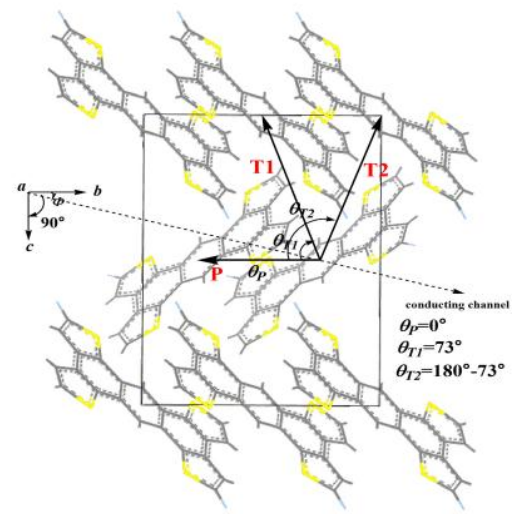

(a)

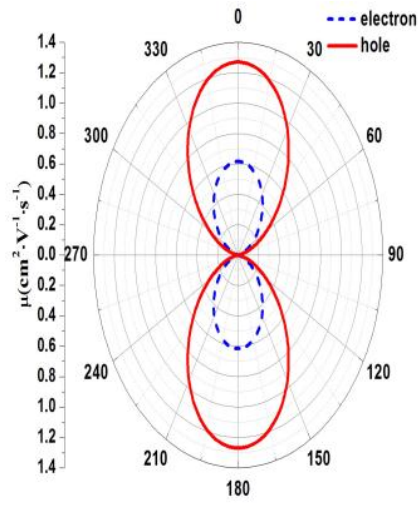

(b)

Figure 7. (a) Illustration of projecting different hopping pathways to a transistor channel in the $b$-c plane of ATT2 crystal; $\theta_{\mathrm{P}}, \theta_{\mathrm{T} 1}$ and $\theta_{\mathrm{T} 2}$ are the angles of $P, T_{1}$ and $T_{2}$ dimers relative to the reference crystallographic axis $b ; \Phi$ is the angle of a transistor channel relative to the reference crystallographic axis $b$. (b) The simulated hole (red) and electron (blue) anisotropic mobilities in the $b$-c plane of ATT2. 


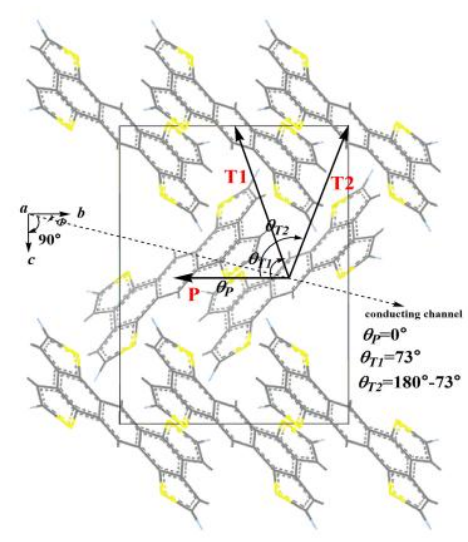

(a)

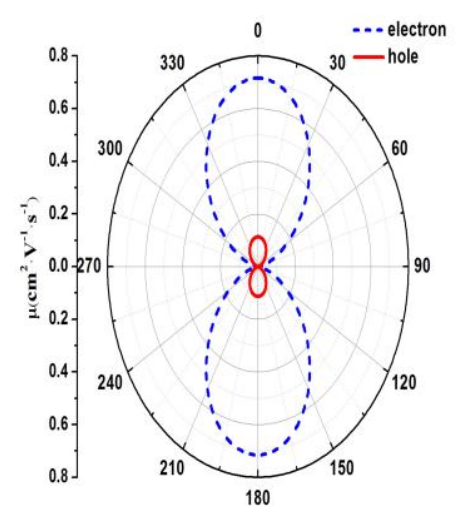

(b)

Figure 8. (a) Illustration of projecting different hopping pathways to a transistor channel in the $b$-c plane of ATT3 crystal; $\theta_{\mathrm{P}}, \theta_{\mathrm{T} 1}$ and $\theta_{\mathrm{T} 2}$ are the angles of $P, T_{1}$ and $T_{2}$ dimers relative to the reference crystallographic axis $b ; \Phi$ is the angle of a transistor channel relative to the reference crystallographic axis $b$. (b) The simulated hole (red) and electron (blue) anisotropic mobilities in the $b$-c plane of ATT3. 


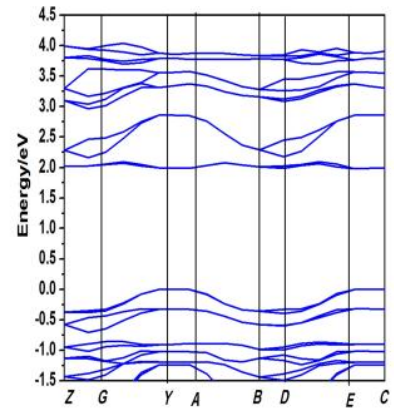

ATT

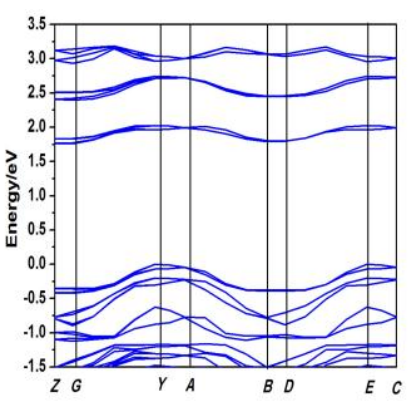

ATT2

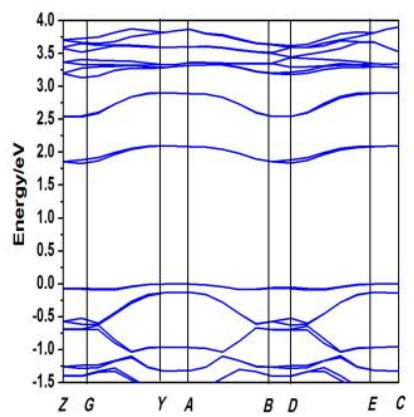

ATT1

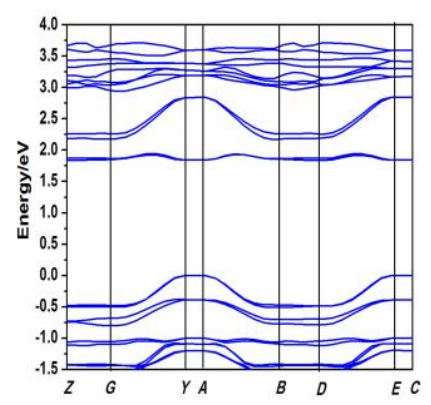

ATT3

Figure 9. The band structures of the crystals ATT, ATT1, ATT2 and ATT3. High symmetry k-points in the first Brillouin zone are $Z=(0,0,0.5), G=(0,0,0), Y=(0,0.5,0), A=(-0.5,0.5,0), B=(-0.5,0,0)$, $D=(-0.5,0,0.5), E=(-0.5,0.5,0.5), C=(0,0.5,0.5)$. 
Table 1. The reorganization energies for hole and electron transfer $\left(\lambda_{\mathrm{h}}\right.$ and $\left.\lambda_{\mathrm{e}}\right)$ of the ATT, ATT1, ATT2 and ATT3 are calculated by adiabatic potential surfaces (AP) using the B3LYP/6-31G(d,p) method. All in $\mathrm{eV}$.

\begin{tabular}{ccc}
\hline Compound & $\lambda_{\mathrm{h}}$ & $\lambda_{\mathrm{e}}$ \\
\hline ATT & 0.124 & 0.149 \\
ATT1 & 0.143 & 0.159 \\
ATT2 & 0.176 & 0.138 \\
ATT3 & 0.162 & 0.177 \\
\hline
\end{tabular}


Table 2. The calculated ionization potentials and electron affinities for adiabatic and vertical states of the ATT, ATT1, ATT2 and ATT3 (all in eV) at the B3LYP/6-31G(d,p) level

\begin{tabular}{ccccc}
\hline Compound & $I P_{a}$ & $I P_{V}$ & $E A_{a}$ & $E A_{V}$ \\
\hline ATT & 6.564 & 6.502 & 0.517 & 0.587 \\
ATT1 & 6.659 & 6.588 & 0.680 & 0.759 \\
ATT2 & 6.647 & 6.562 & 0.678 & 0.757 \\
ATT3 & 6.752 & 6.671 & 0.839 & 0.927 \\
\hline
\end{tabular}


Table 3. The calculated hole $\left(V_{h}\right)$ and electron $\left(V_{e}\right)$ transfer integral (meV) and the mass center distances $d(\AA)$ for compounds ATT, ATT1, ATT2 and ATT3 along different hopping pathways at the PW91PW91/6-31G(d,p) level

\begin{tabular}{|c|c|c|c|c|c|c|c|c|c|c|c|c|}
\hline \multirow[b]{2}{*}{ Pathways } & \multicolumn{3}{|c|}{ ATT } & \multicolumn{3}{|c|}{ ATT1 } & \multicolumn{3}{|c|}{ ATT2 } & \multicolumn{3}{|c|}{ ATT3 } \\
\hline & $V_{h}$ & $V_{e}$ & $d$ & $V_{h}$ & $V_{e}$ & $d$ & $V_{h}$ & $V_{e}$ & $d$ & $V_{h}$ & $V_{e}$ & $d$ \\
\hline $\mathrm{P} 1$ & -70.51 & 44.01 & 5.03 & -127.43 & 13.90 & 4.74 & -96.31 & -60.00 & 4.45 & -30.30 & -83.81 & 3.83 \\
\hline $\mathrm{P} 2$ & -70.51 & 44.01 & 5.03 & -127.43 & 13.90 & 4.74 & -96.31 & -60.00 & 4.45 & -30.30 & -83.81 & 3.83 \\
\hline P3 & 0.70 & 5.60 & 9.60 & 1.00 & 2.60 & 9.57 & -0.50 & -2.30 & 9.70 & -0.60 & 0.40 & 11.71 \\
\hline $\mathrm{P} 4$ & 0.70 & 5.60 & 9.60 & 1.00 & 2.60 & 9.57 & -0.50 & -2.30 & 9.70 & -0.60 & 0.40 & 11.71 \\
\hline P5 & 0.70 & 5.60 & 9.60 & 1.00 & 2.60 & 9.57 & -0.50 & -2.30 & 9.70 & -0.60 & 0.40 & 11.71 \\
\hline P6 & 0.70 & 5.60 & 9.60 & 1.00 & 2.60 & 9.57 & -0.50 & -2.30 & 9.70 & -0.60 & 0.40 & 11.71 \\
\hline P7 & 0.60 & 1.10 & 11.28 & 0.10 & 0.30 & 13.03 & 9.40 & 5.70 & 11.24 & -1.00 & -1.70 & 13.48 \\
\hline P8 & 1.30 & -1.00 & 12.35 & 0.10 & 0.30 & 13.03 & 9.40 & 5.70 & 11.24 & -1.00 & -1.70 & 13.48 \\
\hline P9 & & & & -0.10 & 0.40 & 12.14 & -0.20 & -0.30 & 10.33 & -0.10 & 0.6 & 12.92 \\
\hline P10 & & & & -0.10 & 0.40 & 12.14 & -0.20 & -0.30 & 10.33 & -0.10 & 0.6 & 12.92 \\
\hline
\end{tabular}


Table 4. The calculated carrier mobilities $\left(\mathrm{cm}^{2} \cdot \mathrm{V}^{-1} \cdot \mathrm{s}^{-1}\right)$ for both hole and electron of ATT, ATT1, ATT2 and ATT3

\begin{tabular}{ccc}
\hline Compound & $\mu_{h}$ & $\mu_{e}$ \\
\hline ATT & $1.16(0.012)^{\mathrm{a}}$ & 0.36 \\
ATT1 & 2.59 & 0.02 \\
ATT2 & 0.85 & 0.41 \\
ATT3 & 0.08 & 0.48 \\
\hline
\end{tabular}

a The value in the parentheses is the experimental hole mobility, measured based on film in Ref. [6]. 
Graphic abstract

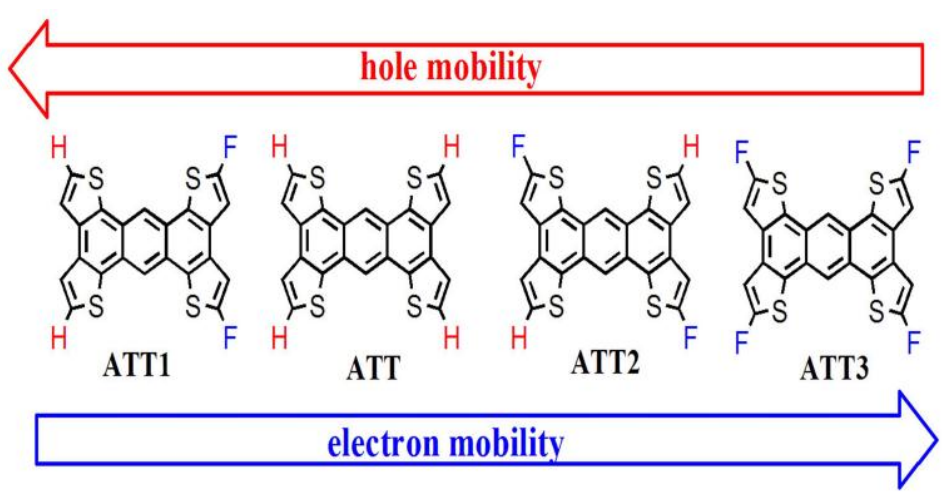

